\title{
Zagreb Topological indices for hexadentate 3- hydroxypyridinones-terminated dendrimers used in iron binding and anti-microbial activities
}

\author{
Thayamathy Pio Jude ${ }^{1}$, Elango Panchadcharam ${ }^{1 *}$, Koneswaran Masilamani ${ }^{2}$ \\ ${ }^{1}$ Department of Mathematics, Faculty of Science, Eastern University, Sri Lanka, Sri Lanka. \\ ${ }^{2}$ Department of Chemistry, Faculty of Science, Eastern University, Sri Lanka, Sri Lanka. \\ *Correspondence: elangop@esn.ac.lk; (D) https://orcid.org/0000-0002-7359-989X
}

Received: 03 ${ }^{\text {rd }}$ March 2019, Revised: $29^{\text {th }}$ May 2020, Accepted: $25^{\text {th }}$ June 2020

\begin{abstract}
Among the degree based topological indices which are mostly used in the study of chemical graph theory, the Zagreb index plays a prominent role in the study of therapies and treatments. There were different versions of the Zagreb indices defined in the literature and used for different purposes. In this paper, we calculated the different versions of the Zagreb indices for the hexadentate 3hydroxypyridinones-terminated dendrimers which are used in iron binding and anti-microbial activities. These indices may play an important role in determining properties of these compounds.
\end{abstract}

Keywords: Anti-microbial activities, dendrimers, iron binding, topological index, Zagreb index.

\section{Introduction}

Iron performs a crucial role in the growth and development of living systems. Therefore, it is one of the most vital trace elements in biological systems (Weizman et al. 1996). Iron is an essential element in hemoglobin, myoglobin, cytochromes and important in many biological processes (Haas and Browlie 2001, Zhang and Enns 2008, Evstatiev et al. 2012). However, both iron deficiency and the excessive iron in the body are harmful to human health. Excessive iron in the body is usually removed by iron-specific chelators (Saliba et al. 2016). Of the few iron specific chelators, macromolecular iron chelators play a major role in the treatment in removing both chronic and acute excessive iron in the body (Mahoney et al. 1989). Dendrimers are a class of synthetic macromolecules with highly branched structures which built up by repeating the assembly of the constituent layers or generations. These structures have a central core and different functional groups attached to their outer surface (Zhu and Shi 2013). These dendrimer structures are used as iron chelators for therapeutic purposes. These iron chelators bind to the iron and to the complex which are kinetically inert and non-toxic. Furthermore, these complexes are not absorbed by the 
gastrointestinal tract of the human due to their large size so will be excreted from the human body. Furthermore, the macromolecular iron chelators inhibit the growth of bacteria hence are used in the treatment of wound healings (Zhou et al. 2011). Zhou et. al synthesized hexadentate 3-hydroxypyridinones-terminated dendrimers that could be used for iron bindings and anti-bacterial purposes (Zhou et al. 2018).

Chemical graph theory is an important tool for studying molecular structures. In theoretical chemistry, molecular descriptors, especially topological indices are used for modelling information of molecules which are used in biology and pharmacology. Therefore, determining topological indices of dendrimers will give a suitable correlation between chemical structure and its activity (Farahani et al. 2015, Soleimani et al. 2016). In this work, different versions of the Zagreb indices are calculated for the hexadentate 3-hydroxypyridinones-terminated dendrimers which are used in iron binding and anti-microbial activities.

A molecular graph is a simple graph which has neither loops nor multiple edges. The atoms and the chemical bonds between them in molecular graphs, are represented by vertices and edges respectively in the graph theory. A graph $G=G(V, E)$ is a pair of nonempty set of vertices $V=V(G)$ and the set of connected edges $E=E(G)$ if there exists a connection between any pair of vertices in $G$. The degree $d_{v}(G)$ or $d_{v}$ of a vertex $v$ in the set of vertices $V(G)$ in a connected graph $G$ is the number of vertices which are connected to that vertex by the edges. The concept of degree is closely related to the concept of valence bond in chemistry (Gutman 2013).

A topological index is a numerical quantity which is derived mathematically in a direct and unambiguous manner from the structural graph of a molecule. The topological index is defined to be a function Top: $\mathrm{G}(\mathrm{V}, \mathrm{E}) \rightarrow R$, where $R$ is the set of real numbers. If two graphs are isomorphic then their topological indices are same. Many properties of a chemical compound are closely related to some of the topological indices of its molecular graph. The Wiener index is the first and the most studied distance-based topological index in chemical graph theory. This index was introduced by the chemist H. Wiener (Wiener 1947) in 1947 to demonstrate the correlations between physicochemical properties of organic compounds and the topological structure of their molecular graphs. The Wiener index was defined as the sum of distances between all the carbon atoms in the molecules, in terms of carbon-carbon bonds.

Among the different types of topological indices, the degree-based topological indices are the mostly studied type of topological indices, which play a prominent role in chemical graph theory. One of the oldest degree-based topological indices is the well-known Zagreb index which was introduced by Gutman and Trinajstić (1972) during the analysis of the structure-dependency of total $\pi$-electron energy. Currently researchers are interested in calculating the Zagreb indices for different types of molecular structures which are used in various applications (Aslam et al. 2017, Kana et al. 2018, Jude et al. 2019). 


\section{Methods}

In this paper we considered four types of the hexadentate 3-hydroxypyridinonesterminated dendrimers which are used in iron binding and anti-microbial activities. Zhou et al. 2018 shows that the convergent synthesis of a range of novel hexadentate 3-hydroxypyridinone-terminated dendrimers. Initially, first generation dendrimeric chelators were synthesized and as its structure shown in Figure 1 which contain three hexadentate moieties. The second generation of this dendrimeric chelators was synthesised by the same authors and its structure is shown in Figure 2. Second generation dendrimer was respectively conjugated with di-acid and tri-acid to form protected structures in Figure 3 and Figure 4 which contain six and nine hexadentate centres respectively. We calculated four different versions of the Zagreb indices using the following formulas.

The first Zagreb index (Gutman and Trinajstić 1972) was defined as

$$
\mathrm{M}_{1}(\mathrm{G})=\sum_{\mathrm{v} \in \mathrm{V}(\mathrm{G})}\left(\mathrm{d}_{\mathrm{v}}\right)^{2}=\sum_{\mathrm{uv} \in \mathrm{E}(\mathrm{G})}\left(\mathrm{d}_{\mathrm{u}}+\mathrm{d}_{\mathrm{v}}\right)
$$

and the second Zagreb index (Gutman and Trinajstić 1972) was defined as

$$
M_{2}(G)=\sum_{u v \in E(G)} d_{u} d_{v}
$$

The second modified Zagreb index (Hao 2011) was defined as

$$
m_{M_{2}}(G)=\sum_{u v \in E(G)} \frac{1}{d_{u} d_{v}}
$$

The reduced second Zagreb index which appeared in the literature earlier but so far not been studied in mathematical chemistry was re-presented in the paper (Gutman et al. 2015) with its main mathematical properties. This was defined as

$$
\operatorname{RM}_{2}(G)=\sum_{u v \in E(G)}\left(d_{u}-1\right)\left(d_{v}-1\right)
$$

This is of the difference between the two Zagreb indices $M_{1}(G)$ and $M_{2}(G)$ and if the graph $G$ is a tree, then this is equal to the number of pairs of vertices at distance 3 , which is often referred to as the "Wiener polarity index" in mathematical chemistry (Gutman et al. 2014).

\section{Results and Discussion}

By observing the structure of synthetic route of the first generation dendrimeric chelators, we inferred six partitions of the edge set as: 


$$
\begin{aligned}
& E_{1}(G)=\left\{e=u v \in E(G): d_{u}=1 \text { and } d_{v}=2\right\}, \\
& E_{2}(G)=\left\{e=u v \in E(G): d_{u}=1 \text { and } d_{v}=3\right\}, \\
& E_{3}(G)=\left\{e=u v \in E(G): d_{u}=d_{v}=2\right\}, \\
& E_{4}(G)=\left\{e=u v \in E(G): d_{u}=2 \text { and } d_{v}=3\right\}, \\
& E_{5}(G)=\left\{e=u v \in E(G): d_{u}=2 \text { and } d_{v}=4\right\}, \\
& E_{6}(G)=\left\{e=u v \in E(G): d_{u}=d_{v}=3\right\} .
\end{aligned}
$$

Also, we get

$\left|E_{1}(G)\right|=9,\left|E_{2}(G)\right|=33,\left|E_{3}(G)\right|=42,\left|E_{4}(G)\right|=69,\left|E_{5}(G)\right|=12,\left|E_{6}(G)\right|=27$

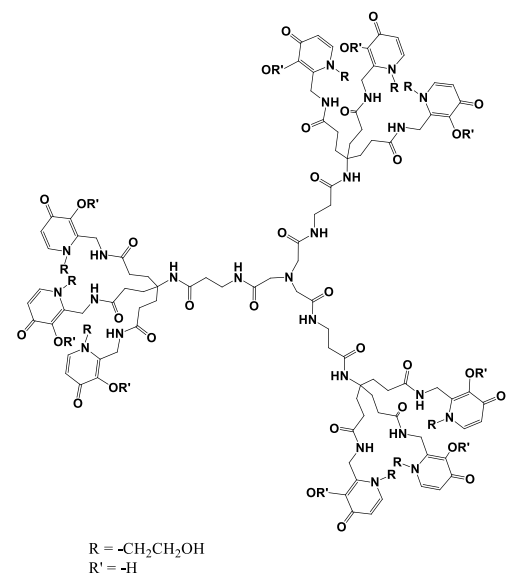

Fig. 1. Structure of the first generation dendrimeric chelators (Zhou et al. 2018)

\section{Theorem 1.}

Let $\mathrm{G}$ be the first generation dendrimeric chelators. Then the first Zagreb index $M_{1}(G)$, the second Zagreb index $M_{2}(G)$, the second modified Zagreb index ${ }^{m} M_{2}(G)$ and the reduced second Zagreb index $R M_{2}(G)$ for $\mathrm{G}$ are
1. $M_{1}(G)=906$
2. $M_{2}(G)=1038$
3. ${ }^{m} M_{2}(G)=42$
4. $\quad R M_{2}(G)=324$ 


\section{Proof:}

Using this edge partition of the first generation dendrimeric chelators and by the respective formulas of different versions of the Zagreb indices, we get

1. $M_{1}(G)=\sum_{u v \in E(G)}\left(d_{u}+d_{v}\right)$

$$
\begin{gathered}
=\left|E_{1}(G)\right|(1+2)+\left|E_{2}(G)\right|(1+3)+\left|E_{3}(G)\right|(2+2)+\left|E_{4}(G)\right|(2+3) \\
\quad+\left|E_{5}(G)\right|(2+4)+\left|E_{6}(G)\right|(3+3) \\
=9 \times 3+33 \times 4+42 \times 4+69 \times 5+12 \times 6+27 \times 6=906
\end{gathered}
$$

2. $M_{2}(G)=\sum_{u v \in E(G)}\left(d_{u} \times d_{v}\right)$

$$
\begin{gathered}
=\left|E_{1}(G)\right|(1 \times 2)+\left|E_{2}(G)\right|(1 \times 3)+\left|E_{3}(G)\right|(2 \times 2)+\left|E_{4}(G)\right|(2 \times 3) \\
\quad+\left|E_{5}(G)\right|(2 \times 4)+\left|E_{6}(G)\right|(3 \times 3) \\
=9 \times 2+33 \times 3+42 \times 4+69 \times 6+12 \times 8+27 \times 9=1038
\end{gathered}
$$

3. ${ }^{m} M_{2}(G)=\sum_{u v \in E(G)} \frac{1}{d_{u} d_{v}}$

$$
\begin{aligned}
=\left|E_{1}(G)\right| & \frac{1}{1 \times 2}+\left|E_{2}(G)\right| \frac{1}{1 \times 3}+\left|E_{3}(G)\right| \frac{1}{2 \times 2}+\left|E_{4}(G)\right| \frac{1}{2 \times 3}+\left|E_{5}(G)\right| \frac{1}{2 \times 4} \\
& +\left|E_{6}(G)\right| \frac{1}{3 \times 3} \\
= & \frac{9}{2}+\frac{33}{3}+\frac{42}{4}+\frac{69}{6}+\frac{12}{8}+\frac{27}{9}=42
\end{aligned}
$$

4. $\quad R M_{2}(G)=\sum_{u v \in E(G)}\left(d_{u}-1\right)\left(d_{v}-1\right)$

$$
\begin{gathered}
=\left|E_{1}(G)\right|(1-1)(2-1)+\left|E_{2}(G)\right|(1-1)(3-1)+\left|E_{3}(G)\right|(2-1)(2-1) \\
+\left|E_{4}(G)\right|(2-1)(3-1)+\left|E_{6}(G)\right|(2-1)(4-1) \\
\quad+\left|E_{5}(G)\right|(3-1)(3-1) \\
=9 \times 0+33 \times 0+42+69 \times 2+12 \times 3+27 \times 4=324
\end{gathered}
$$

\section{Remark:}

By observing the structure of the second generation dendrimeric chelators, we inferred six partitions of the edge set as:

$$
\begin{aligned}
& E_{1}(G)=\left\{e=u v \in E(G): d_{u}=1 \text { and } d_{v}=2\right\} \\
& E_{2}(G)=\left\{e=u v \in E(G): d_{u}=1 \text { and } d_{v}=3\right\} \\
& E_{3}(G)=\left\{e=u v \in E(G): d_{u}=2 \text { and } d_{v}=3\right\} \\
& E_{4}(G)=\left\{e=u v \in E(G): d_{u}=d_{v}=2\right\} \\
& E_{5}(G)=\left\{e=u v \in E(G): d_{u}=2 \text { and } d_{v}=4\right\} \\
& E_{6}(G)=\left\{e=u v \in E(G): d_{u}=d_{v}=3\right\}
\end{aligned}
$$


Also, we get

$\left|E_{1}(G)\right|=10,\left|E_{2}(G)\right|=25,\left|E_{3}(G)\right|=100,\left|E_{4}(G)\right|=95,\left|E_{5}(G)\right|=16,\left|E_{6}(G)\right|=27$

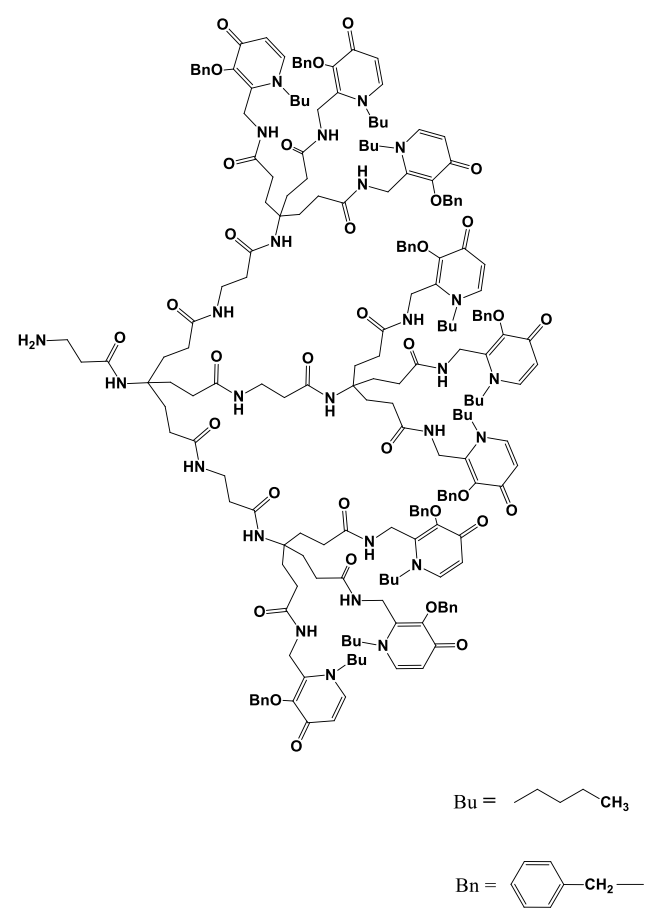

Fig. 2: Second generation dendrimeric chelators (Zhou et al. 2018).

\section{Theorem 2.}

Let $G$ be the second generation dendrimeric chelators. Then the first Zagreb index $M_{1}(G)$, the second Zagreb index $M_{2}(G)$, the second modified Zagreb index ${ }^{m} M_{2}(G)$ and the reduced second Zagreb index $R M_{2}(G)$ for $G$ are
1. $M_{1}(G)=1263$
2. $M_{2}(G)=1436$
3. ${ }^{m} M_{2}(G)=\frac{355}{6}$
4. $R M_{2}(G)=446$

\section{Proof:}

Using this edge partition of the second generation dendrimeric chelators and by the respective formulas of different versions of the Zagreb indices, we get 
1. $\quad M_{1}(G)=\sum_{u v \in E(G)}\left(d_{u}+d_{v}\right)$

$$
\begin{gathered}
=\left|E_{1}(G)\right|(1+2)+\left|E_{2}(G)\right|(1+3)+\left|E_{3}(G)\right|(2+2)+\left|E_{4}(G)\right|(2+3) \\
\quad+\left|E_{5}(G)\right|(2+4)+\left|E_{6}(G)\right|(3+3) \\
=10 \times 3+25 \times 4+100 \times 4+95 \times 5+16 \times 6+27 \times 6=1263
\end{gathered}
$$

2. $M_{2}(G)=\sum_{u v \in E(G)}\left(d_{u} \times d_{v}\right)$

$$
\begin{aligned}
=\left|E_{1}(G)\right| & (1 \times 2)+\left|E_{2}(G)\right|(1 \times 3)+\left|E_{3}(G)\right|(2 \times 2)+\left|E_{4}(G)\right|(2 \times 3) \\
& +\left|E_{5}(G)\right|(2 \times 4)+\left|E_{6}(G)\right|(3 \times 3)
\end{aligned}
$$

$=10 \times 2+25 \times 3+100 \times 4+95 \times 6+16 \times 8+27 \times 9=1436$

3. ${ }^{m} M_{2}(G)=\sum_{u v \in E(G)} \frac{1}{d_{u} d_{v}}$

$$
\begin{aligned}
=\left|E_{1}(G)\right| & \frac{1}{1 \times 2}+\left|E_{2}(G)\right| \frac{1}{1 \times 3}+\left|E_{3}(G)\right| \frac{1}{2 \times 2}+\left|E_{4}(G)\right| \frac{1}{2 \times 3}+\left|E_{5}(G)\right| \frac{1}{2 \times 4} \\
& +\left|E_{6}(G)\right| \frac{1}{3 \times 3} \\
= & \frac{10}{2}+\frac{25}{3}+\frac{100}{4}+\frac{95}{6}+\frac{16}{8}+\frac{27}{9}=\frac{355}{6}
\end{aligned}
$$

4. $R M_{2}(G)=\sum_{u v \in E(G)}\left(d_{u}-1\right)\left(d_{v}-1\right)$

$$
\begin{aligned}
& =\left|E_{1}(G)\right|(1-1)(2-1)+\left|E_{2}(G)\right|(1-1)(3-1)+\left|E_{3}(G)\right|(2-1)(2-1) \\
& \quad+\left|E_{4}(G)\right|(2-1)(3-1)+\left|E_{6}(G)\right|(2-1)(4-1) \\
& \quad+\left|E_{5}(G)\right|(3-1)(3-1) \\
& =10 \times 0+25 \times 0+100+95 \times 2+16 \times 3+27 \times 4=446
\end{aligned}
$$

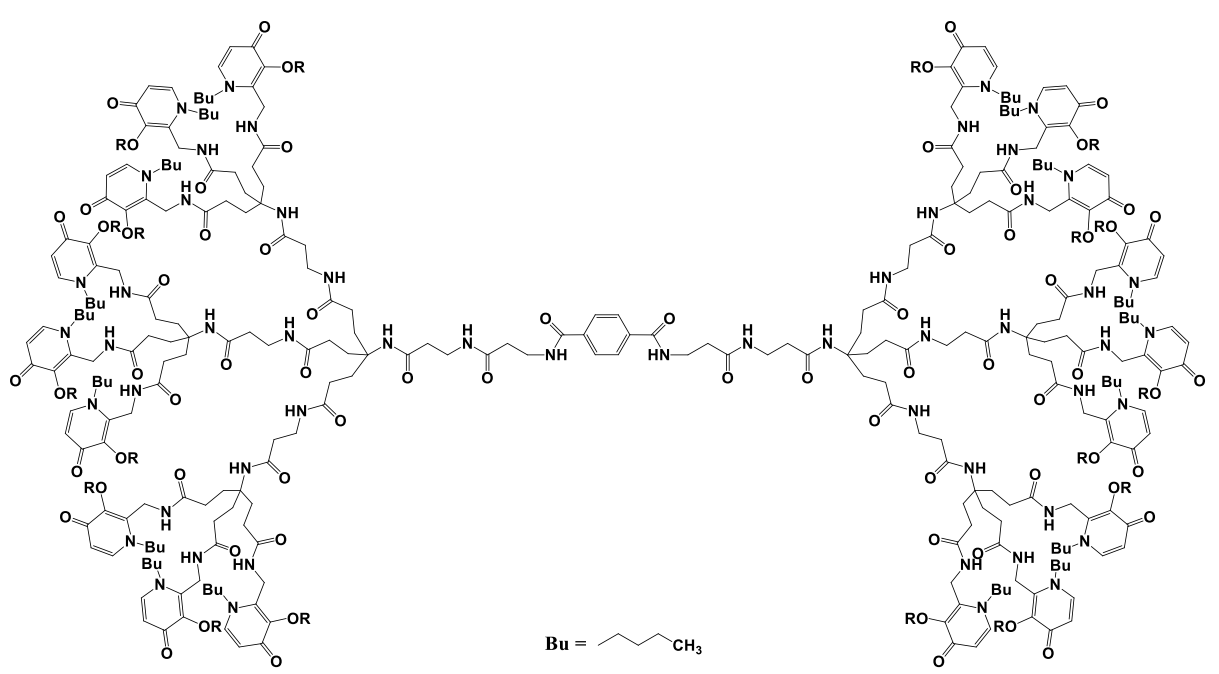

Fig. 3. Dendrimeric chelators containing six hexadentate centres (Zhou et al. 2018) 
By observing the structure of dendrimeric chelators contain six hexadentate centres, we inferred six partitions of the edge set as:

$$
\begin{aligned}
& E_{1}(G)=\left\{e=u v \in E(G): d_{u}=1 \text { and } d_{v}=2\right\}, \\
& E_{2}(G)=\left\{e=u v \in E(G): d_{u}=1 \text { and } d_{v}=3\right\} \\
& E_{3}(G)=\left\{e=u v \in E(G): d_{u}=d_{v}=2\right\}, \\
& E_{4}(G)=\left\{e=u v \in E(G): d_{u}=2 \text { and } d_{v}=3\right\} \\
& E_{5}(G)=\left\{e=u v \in E(G): d_{u}=2 \text { and } d_{v}=4\right\}, \\
& E_{6}(G)=\left\{e=u v \in E(G): d_{u}=d_{v}=3\right\} .
\end{aligned}
$$

Also, we get

$\left|E_{1}(G)\right|=18,\left|E_{2}(G)\right|=72,\left|E_{3}(G)\right|=118,\left|E_{4}(G)\right|=146,\left|E_{5}(G)\right|=32,\left|E_{6}(G)\right|=56$.

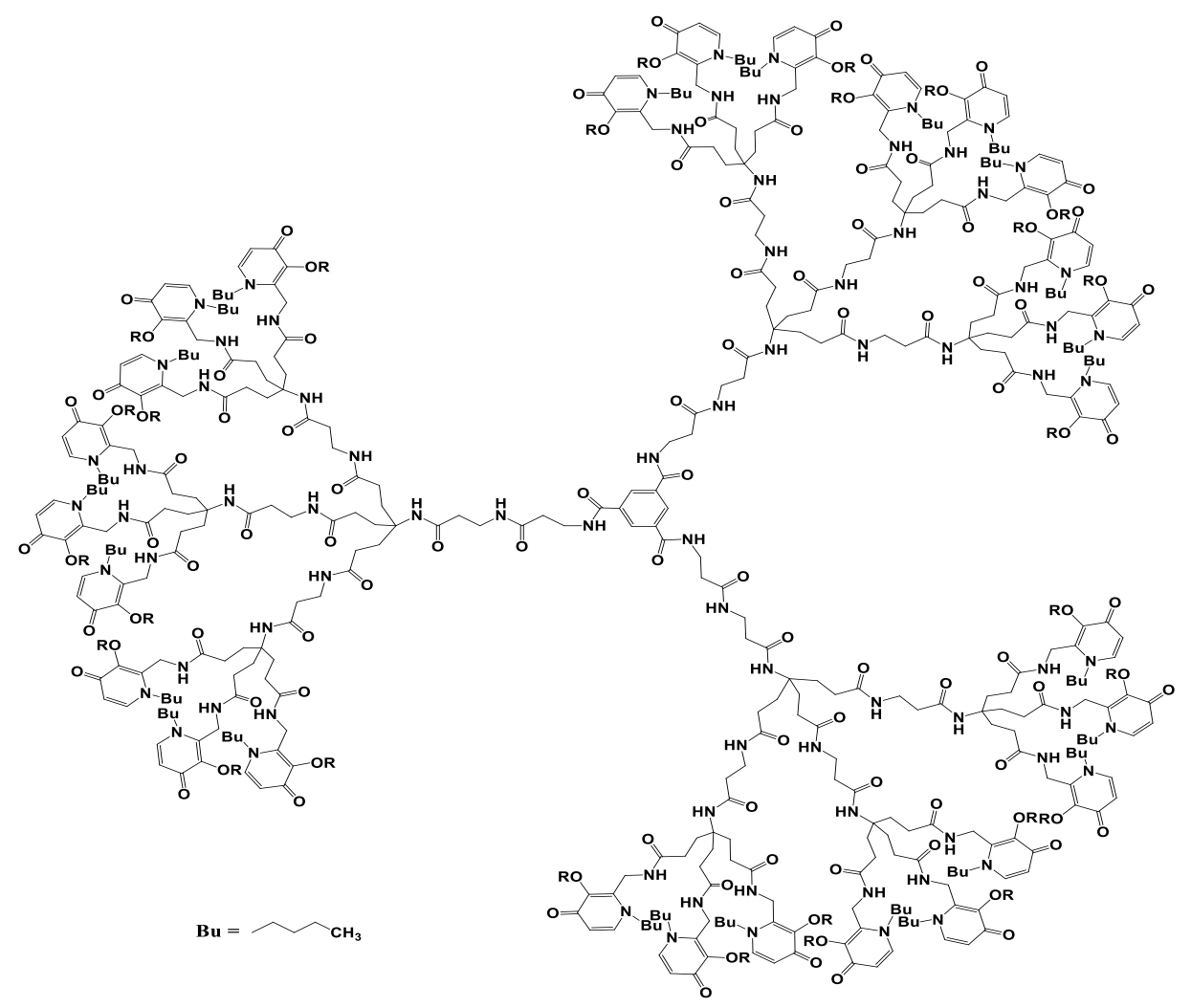

Fig. 4. Dendrimeric chelators containing nine hexadentate centres (Zhou et al. 2018) 


\section{Theorem 3.}

Let $G$ be the dendrimeric chelators contain six hexadentate centres. Then the first Zagreb index $M_{1}(G)$, the second Zagreb index $M_{2}(G)$, the second modified Zagreb index ${ }^{m} M_{2}(G)$ and the reduced second Zagreb index $R M_{2}(G)$ for $G$ are
1. $\quad M_{1}(G)=2072$
2. $M_{2}(G)=2360$
3. ${ }^{m} M_{2}(G)=\frac{1747}{18}$
4. $R M_{2}(G)=730$

\section{Proof:}

Using this edge partition of the dendrimeric chelators contain six hexadentate centres and by the respective formulas of different versions of the Zagreb indices, we get

$$
\begin{aligned}
& \text { 1. } M_{1}(G)=\sum_{u v \in E(G)}\left(d_{u}+d_{v}\right) \\
& =\left|E_{1}(G)\right|(1+2)+\left|E_{2}(G)\right|(1+3)+\left|E_{3}(G)\right|(2+2)+\left|E_{4}(G)\right|(2+3) \\
& +\left|E_{5}(G)\right|(2+4)+\left|E_{6}(G)\right|(3+3) \\
& =18 \times 3+72 \times 4+118 \times 4+146 \times 5+32 \times 6+56 \times 6=2072 \\
& \text { 2. } M_{2}(G)=\sum_{u v \in E(G)}\left(d_{u} \times d_{v}\right) \\
& =\left|E_{1}(G)\right|(1 \times 2)+\left|E_{2}(G)\right|(1 \times 3)+\left|E_{3}(G)\right|(2 \times 2)+\left|E_{4}(G)\right|(2 \times 3) \\
& +\left|E_{5}(G)\right|(2 \times 4)+\left|E_{6}(G)\right|(3 \times 3) \\
& =18 \times 2+72 \times 3+118 \times 4+146 \times 6+32 \times 8+56 \times 9=2360 \\
& =\left|E_{1}(G)\right| \frac{1}{1 \times 2}+\left|E_{2}(G)\right| \frac{1}{1 \times 3}+\left|E_{3}(G)\right| \frac{1}{2 \times 2}+\left|E_{4}(G)\right| \frac{1}{2 \times 3}+\left|E_{5}(G)\right| \frac{1}{2 \times 4} \\
& +\left|E_{6}(G)\right| \frac{1}{3 \times 3} \\
& =\frac{18}{2}+\frac{72}{3}+\frac{118}{4}+\frac{146}{6}+\frac{32}{8}+\frac{56}{9}=\frac{1747}{18} \\
& =\left|E_{1}(G)\right|(1-1)(2-1)+\left|E_{2}(G)\right|(1-1)(3-1)+\left|E_{3}(G)\right|(2-1)(2-1) \\
& +\left|E_{4}(G)\right|(2-1)(3-1)+\left|E_{6}(G)\right|(2-1)(4-1)+\left|E_{5}(G)\right|(3-1)(3-1) \\
& =18 \times 0+72 \times 0+118+146 \times 2+32 \times 3+56 \times 4=730
\end{aligned}
$$




\section{Remark}

By observing the structure of dendrimeric chelators contain nine hexadentate centres, we inferred six partitions of the edge set as:

$$
\begin{aligned}
& E_{1}(G)=\left\{e=u v \in E(G): d_{u}=1 \text { and } d_{v}=2\right\}, \\
& E_{2}(G)=\left\{e=u v \in E(G): d_{u}=1 \text { and } d_{v}=3\right\} \\
& E_{3}(G)=\left\{e=u v \in E(G): d_{u}=d_{v}=2\right\}, \\
& E_{4}(G)=\left\{e=u v \in E(G): d_{u}=2 \text { and } d_{v}=3\right\} \\
& E_{5}(G)=\left\{e=u v \in E(G): d_{u}=2 \text { and } d_{v}=4\right\}, \\
& E_{6}(G)=\left\{e=u v \in E(G): d_{u}=d_{v}=3\right\} .
\end{aligned}
$$

Also, we get

$\left|E_{1}(G)\right|=27,\left|E_{2}(G)\right|=108,\left|E_{3}(G)\right|=174,\left|E_{4}(G)\right|=219,\left|E_{5}(G)\right|=48,\left|E_{6}(G)\right|=84$.

\section{Theorem 4.}

Let $G$ be the dendrimeric chelators contain nine hexadentate centres. Then the first Zagreb index $M_{1}(G)$, the second Zagreb index $M_{2}(G)$, the second modified Zagreb index ${ }^{m} M_{2}(G)$ and the reduced second Zagreb index $R M_{2}(G)$ for $G$ are

1. $M_{1}(G)=3096$

2. $M_{2}(G)=3432$

3. ${ }^{m} M_{2}(G)=\frac{869}{6}$

4. $R M_{2}(G)=1092$

\section{Proof:}

Using this edge partition of the dendrimeric chelators contain nine hexadentate centres and by the respective formulas of different versions of the Zagreb indices, we get

1. $M_{1}(G)=\sum_{u v \in E(G)}\left(d_{u}+d_{v}\right)$

$$
\begin{gathered}
=\left|E_{1}(G)\right|(1+2)+\left|E_{2}(G)\right|(1+3)+\left|E_{3}(G)\right|(2+2)+\left|E_{4}(G)\right|(2+3) \\
\quad+\left|E_{5}(G)\right|(2+4)+\left|E_{6}(G)\right|(3+3) \\
=27 \times 3+108 \times 4+174 \times 4+219 \times 5+48 \times 6+84 \times 6=3096
\end{gathered}
$$

2. $M_{2}(G)=\sum_{u v \in E(G)}\left(d_{u} \times d_{v}\right)$ 


$$
\begin{aligned}
& =\left|E_{1}(G)\right|(1 \times 2)+\left|E_{2}(G)\right|(1 \times 3)+\left|E_{3}(G)\right|(2 \times 2)+\left|E_{4}(G)\right|(2 \times 3) \\
& \quad+\left|E_{5}(G)\right|(2 \times 4)+\left|E_{6}(G)\right|(3 \times 3) \\
& =27 \times 2+108 \times 3+174 \times 4+219 \times 6+48 \times 8+84 \times 9=3432
\end{aligned}
$$

3. ${ }^{m} M_{2}(G)=\sum_{u v \in E(G)} \frac{1}{d_{u} d_{v}}$

$$
\begin{aligned}
=\left|E_{1}(G)\right| & \frac{1}{1 \times 2}+\left|E_{2}(G)\right| \frac{1}{1 \times 3}+\left|E_{3}(G)\right| \frac{1}{2 \times 2}+\left|E_{4}(G)\right| \frac{1}{2 \times 3}+\left|E_{5}(G)\right| \frac{1}{2 \times 4} \\
& +\left|E_{6}(G)\right| \frac{1}{3 \times 3} \\
= & \frac{27}{2}+\frac{108}{3}+\frac{174}{4}+\frac{219}{6}+\frac{48}{8}+\frac{84}{9}=\frac{869}{6}
\end{aligned}
$$

4. $R M_{2}(G)=\sum_{u v \in E(G)}\left(d_{u}-1\right)\left(d_{v}-1\right)$

$$
\begin{aligned}
=\left|E_{1}(G)\right| & (1-1)(2-1)+\left|E_{2}(G)\right|(1-1)(3-1)+\left|E_{3}(G)\right|(2-1)(2-1) \\
& +\left|E_{4}(G)\right|(2-1)(3-1)+\left|E_{5}(G)\right|(2-1)(4-1) \\
& ++\left|E_{6}(G)\right|(3-1)(3-1) \\
=27 \times 0 & +108 \times 0+174+219 \times 2+48 \times 3+84 \times 4=1092
\end{aligned}
$$

\section{Conclusions}

In this work, we considered, hexadentate 3-hydroxypyridinones-terminated dendrimers which are used in iron binding and anti-microbial activities. We calculated the four different versions of the Zagreb indices of these four novel hexadentate 3hydroxypyridinone-terminated dendrimers which have been demonstrated to have a high affinity towards iron (III) ions and have the antimicrobial property. These indices are calculated by using the edge set partitions of these structures of dendrimers.

\section{Acknowledgments}

We acknowledge Prof. Robert C. Hider and his research team for granting permission to reproduce the figures in Zhou et al. 2018. Two anonymous reviewers are acknowledged for comments on the initial draft of the manuscript.

\section{References}

Aslam A, Bashir Y, Ahmad S, Gao W. 2017. On Topological Indices of Certain Dendrimer Structures. Zeitschrift für Naturforschung 72: 559-566.

Evstatiev R, Gasche C. 2012. Iron sensing and signalling. Gut 61: 933-952.

Farahani MR., Gao W, Kanna RMR. 2015. The connective eccentric index for an infinite family of dendrimers. Indian Journal of Fundamental and Applied Life Sciences 5 (S4): 766-771.

Gutman I. 2013. Degree-Based Topological Indices. Croatica Chemical Acta 86: 351-361.

Gutman I, Furtula B, Elphick C. 2014. Three New/Old Vertex-Degree-Based Topological Indices. MATCH-Communications in Mathematical and Computational Chemistry 72: 617-632. 
Gutman I, Trinajstić N. 1972. Graph theory and molecular orbitals total f-electron energy of alternant hydrocarbons. Chemical Physics Letters 77: 535-538.

Haas JD, Browlie IV T. 2001. Iron deficiency and reduced work capacity: a critical review of the research to determine a causal relationship. Journal of Nutrition 131: 676S-690S.

Hao J. 2011. Theorems about Zagreb Indices and Modified Zagreb Indices. MATCH-Communications in Mathematical and Computational Chemistry 65: 659-670.

Jude TP, Panchadcharam E, Masilamani K. 2019. Computation of Zagreb and Randić indices of two biodegradable dendrimers used in cancer therapy. Ceylon Journal of Science 48: 359-366.

Kang SM., Zahid MA., Virk AR., Nazeer W, Gao W. 2018. Calculating the Degree-based Topological. Indices of Dendrimers, Open Chemistry 61: 681-688.

Mahoney JR, Hallaway PE, Hedlund BE, Eaton JW. 1989. Acute iron poisoning - Rescue with macromolecular chelators. Journal of Clinical Investigation 84: 1362-1366.

Saliba AN, El Rassi F, Taher AT. 2016. Clinical monitoring and management of complications related to chelation therapy in patients with beta-thalassemia. Expert Rev Hematol. 9, 151-168.

Soleimani N, Mohseni E, Maleki N. 2016. Connectivity indices of some famous dendrimers. Journal of Chemical and Pharmaceutical Research. 8(8): 229-235.

Weizman H, Ardon O, Mester B, Libman J, Dwir O, Hadar Y, Chen Y, Shanzer A. 1996. Fluorescentlylabeled ferrichrome analogs as probes for receptor-mediated, microbial iron uptake. Journal of the American Chemical Society 118: 12368-12375.

Wiener HJ. 1947. Structural Determination of Paraffin Boiling Points, J. Amer. Chem. Soc. 69: 17-20.

Zhang AS, Enns CA. 2008. Iron Homeostasis: Recently Identified Proteins Provide Insight into Novel Control Mechanisms. Journal of Biological Chemistry 284: 711-715.

Zhou T, Chen K, Kong L-M, Liu M-S, Ma Y-M, Xie Y-Y, Hider RC. 2018. Synthesis, iron binding and antimicrobial properties of hexadentate 3-hydroxypyridinones-terminated dendrimers. Bioorganic \& Medicinal Chemistry Letters 28: 2504-2512.

Zhou T, Winkelmann G, Dai ZY, Hider RC. 2011. Design of clinically useful macromolecular iron chelators. Journal of Pharmacy and Pharmacology 63: 893-903.

Zhu J, Shi X. 2013. Dendrimer-based nanodevices for targeted drug delivery applications. Journal of Materials Chemistry B 134: 4199-4211. 